\title{
Abnormal feeding behaviour in spinalised rats is mediated by hypothalamus: Restorative effect of exposure to extremely low frequency magnetic field
}

\author{
S Ambalayam, S Jain and R Mathur
}

\begin{abstract}
Study design: Experimental study.
Objectives: To investigate the role of hypothalamus in abnormal feeding behaviour after spinal cord injury (SCl) and the effect of exposure to extremely low frequency magnetic field (ELF-MF) on it.

Setting: India.

Methods: Male Wistar rats $(n=44)$ were divided into Sham (laminectomy), SCl (complete transection of T13 spinal cord), $\mathrm{SCl}+\mathrm{MF}$ (ELF-MF exposure to SCl rats), VMHL (lesion of ventromedial hypothalamus; VMH), SCl+VMHL (VMHL after SCl) and SCl $+\mathrm{VMHL}+\mathrm{MF}$ (ELF-MF exposure to SCl+VMHL rats) groups. Food intake (FI), water intake (WI), calorie intake (Cl), body weight (BWT), taste preference and sucrose-induced biphasic (SIB) response to noxious stimulus were studied pre and post surgery. Neuronal activity at VMH was assessed by c-Fos immunohistochemistry. The extent of neuronal degeneration and regeneration in spinal cord was assessed microscopically.

Results: Data revealed post-SCI decrease in $\mathrm{FI}, \mathrm{WI}, \mathrm{Cl}$ and $\mathrm{BWT}$, preference for sodium chloride and citric acid, prolonged analgesic phase of SIB and increased c-Fos immunoreactivity in VMH of SCl rats vs Sham rats. VMH lesion increased $\mathrm{FI}, \mathrm{WI}, \mathrm{Cl}, \mathrm{BW}$, preference for sweet tastants and abolished SIB, whereas in $\mathrm{SCl}+\mathrm{VMHL}$ rats it abolished the effects of $\mathrm{SCl}$ on these parameters indicating probable involvement of $\mathrm{VMH}$ in $\mathrm{SCl}$-induced alteration in feeding behaviour. Exposure to $\mathrm{MF}$ improved the study parameters in $\mathrm{SCl}$ rats and reduced the c-Fos immunoreactivity in VMH besides reduction in lesion volume, greater myelination and neuronal regeneration at $\mathrm{SCl}$ site.
\end{abstract}

Conclusion: $\mathrm{SCl}$ influences VMH, leading to alteration in feeding behaviour, which is improved by exposure to ELF-MF. Spinal Cord (2016) 54, 1076-1087; doi:10.1038/sc.2016.32; published online 10 May 2016

\section{INTRODUCTION}

Spinal cord injury (SCI) leads to under nutrition ${ }^{1}$ besides agonising sensorimotor deficits below the level of lesion. ${ }^{2-6}$ In addition, there is a significant decrease in food intake (FI), water intake (WI) and body weight (BWT) after SCI in rats, ${ }^{7,8}$ which is attributed to gastrointestinal dysmotility resulting from gastro-inhibitory signals ${ }^{9}$ and/or delayed emptying. ${ }^{10,11}$ However, the cause for a significant reduction in FI or intestinal motility after SCI is not known, while the supraspinal involvement has not been addressed.

Among other supraspinal structures, ventromedial nucleus of hypothalamus $(\mathrm{VMH})$, the satiety centre has a vital role in the control of FI. ${ }^{12-15}$ Lesion of VMH is characterised by voracious hyperphagia, hyperdipsia, marked obesity and finickiness for high lipid and carbohydrate diets. ${ }^{15-21}$ Previous reports from our laboratory suggest the role of $\mathrm{VMH}$ in pain modulation by palatable food, which is abolished after its lesion. ${ }^{22-26}$ The influence on FI and food-induced pain modulation is mediated by its glucoresponsive neurons, ${ }^{23,27}$ which respond to changes in both glucose concentration and opioids. ${ }^{28}$

We previously reported a significant decrease in brain concentration of 5-HT in a similar rat model of SCI, which may probably contribute towards a decrease in FI, WI and BWT, as a decrease in 5-HT concentration in the hypothalamus is associated with acute food deprivation. ${ }^{29-31}$ Therefore, the literature indicates hypothalamus as the possible site responsible for dysregulation of FI and energy balance after SCI. We, therefore, systematically investigated the probable involvement of $\mathrm{VMH}$ in the dysregulation of FI and related pain responses after SCI. Moreover, there is no available management strategy for it.

Extremely low frequency magnetic field (ELF-MF) has been reported to improve the quality of life after SCI by significantly increasing locomotor recovery, ${ }^{32-35}$ better autonomic control of urinary bladder, improvement in pain status ${ }^{36}$ and gastrointestinal (GI) motility. ${ }^{37-40}$ In addition, there are reports of facilitation of neuroregenerative processes after nerve injury. ${ }^{41,42}$ However, in these studies, there are significant variations in the type, strength and duration of MF besides the animal model of SCI, which leads to difficulty in understanding the underlying mechanism of its beneficial effect. Earlier reports from our laboratory have also documented beneficial effect of ELF-MF ( $50 \mathrm{~Hz}, 17.96 \mu \mathrm{T}, 2 \mathrm{~h}$ per day for 8 weeks) exposure on sensorimotor function, osteoporosis, FI, WI and BWT in the comparable spinal cord-injured rats. ${ }^{7,43,44}$ However, its influence on VMH control on FI and energy balance after SCI is not known.

In the present study, the precise involvement of $\mathrm{VMH}$ in the SCI-induced hypophagia is investigated. Moreover, the study is 
extended to investigate the influence of ELF-MF on it. We have selected various parameters that are specific for the assessment of $\mathrm{VMH}$ functions related to feeding behaviour and food-induced pain modulation.

\section{MATERIALS AND METHODS}

Animals

Approval for the study was obtained by the Ethical Committee of All India Institute of Medical Sciences. Adult male Wistar rats $(n=44)$ weighing 250-300 g were obtained from Experimental Animal Facility of institute and maintained at $24 \pm 2{ }^{\circ} \mathrm{C}$ and $14: 10 \mathrm{~h}$ light-dark cycle. Rats were marked and individually housed in specific pathogen-free conditions with ad libitum access to laboratory food pellets (Ashirwad Industries, Chandigarh, India) and fresh tap water. They were divided into 7 groups-namely, Sham-SCI $(n=7)$, Sham-SCI+Sham-VMHL $(n=6)$, SCI $(n=7)$, VMHL $(n=6)$, SCI+VMHL $(n=6), \mathrm{SCI}+\mathrm{MF}(n=6)$ and SCI+VMHL+MF $(n=6)$ groups. The data of Sham-SCI and Sham-SCI+Sham-VMHL did not vary among themselves and therefore the pooled data are presented.

\section{Surgical procedures}

Spinal cord injury. On the day of surgery, the rat was anaesthetised with a combination of ketamine hydrochloride $\left(60 \mathrm{mg} \mathrm{kg}^{-1}\right.$ BWT) and xylazine (10 $\mathrm{m} \mathrm{kg}^{-1}$ BWT). The supplement dose of ketamine (i.p) was given to maintain the level of anaesthesia, if required. After skin incision and laminectomy, the spinal cord was completely transected at the T13 level with a fine micro scissor, as described by Ichiyama et al. ${ }^{45}$ which was confirmed under a surgical microscope. A 2-mm long sterile, haemostatic, absorbable gelatin base foam (Abgel; Sri Gopal krishna Pvt. Ltd, Mumbai, India) was placed in the gap that was aligned with the rostral and caudal cut ends of the spinal cord. Rats in the Sham-SCI group received only laminectomy without disrupting the dura. The muscles and skin were sutured in layers with silicon thread (Ethicon, Johnson and Johnson Ltd, Mumbai, India) and topical antibiotic cream (Providone iodine, Glide Chem Pvt. Ltd, Rampur Ghat, India) was applied. Intra- and post-operative body temperatures were maintained by placing the rat on a controlled heating pad (CMA-150, CMA Microdialysis, North Chelmsford, MA, USA). Manual expression of urinary bladder was performed thrice daily until spontaneous voiding was achieved. The rat was monitored for urinary tract infection.

$V M H$ lesion. After anaesthetising the rat with ketamine hydrochloride (60 $\mathrm{mg} \mathrm{kg}^{-1}$ BWT) and xylazine $\left(10 \mathrm{mg} \mathrm{kg}^{-1}\right.$ BWT), its head was fixed in stereotaxic apparatus (Stereodrive, Neurostar, Germany). Bilateral electrolytic lesion of VMH (co-ordinates; AP:2.52 mm, L:0.6 mm, DV:9.4 mm) ${ }^{46}$ was performed with the help of concentric bipolar electrode (outer guide cannula of 21-gauge and inner stainless steel wire of 28 -gauge) by applying $2 \mathrm{~mA} \mathrm{D.C.} \mathrm{for}$ $15 \mathrm{~s}$. In Sham VMHL rats, the electrodes were removed after $5 \mathrm{~min}$ of implantation without applying current. Gentamicin cream was applied after suturing the skin and post-surgical recovery was closely monitored.

\section{MF exposure chamber}

The chamber consisted of modified Helmholtz coils mounted on a stand, a movable platform for the rat cage and a current regulator that maintained constant current through the coils. There were two outer coils $(\mathrm{C} 1, \mathrm{C} 4)$ and two inner coils $(\mathrm{C} 2, \mathrm{C} 3)$ with 18 and 8 turns of wire, respectively, wound on circular frame. The coils were connected in series that provided a uniform MF in the central movable platform area where the rats were kept. ${ }^{47}$ Six rats were placed in a specially designed polypropylene cage $(50 \times 20 \times 15 \mathrm{~cm})$ for their simultaneous exposure to MF. This cage was indigenously built and had six compartments. One rat was kept in each compartment. Before exposing the rats, the MF was precisely adjusted to $17.96 \mu \mathrm{T}$ by using a magnetometer (Walker Scientific Inc. Auburn Hills, MI, USA) and the current regulator.

\section{Feeding behaviour}

Food intake, water intake, calorie intake and body weight. The rat was provided with pre-weighed laboratory food pellets and fresh tap water in spill-proof containers. Left over food and water were measured after $24 \mathrm{~h}$. The cumulative FI, WI and calorie intake (CI) for 7 days was considered for analysis. Total CI was calculated by multiplying the calorific value of food given with the amount of food ingested. When sucrose was given as a part of the experiment, its calorific value was also considered and included for the calculation of total CI. The calorific value of laboratory pellets given to the rats was found to be $3.65 \mathrm{kcal} \mathrm{g}^{-1}$. The BWT was recorded (Tula digital Pvt. Ltd, New Delhi, India) weekly.

Taste preference test. 'Two-bottle' preference test was utilised to determine any alteration in palatability. On the day of experiment, in addition to tap water, one of the tastant solutions-namely, sodium chloride $(0.9 \%)$, sucrose $(20 \%)$, saccharine $(0.15 \%)$, citric acid $(1.05 \%)$ and quinine hydrochloride $(0.024 \%)$ - was provided. One tastant per day was tested in a well-hydrated rat, and the amount of solution ingested during $1 \mathrm{~h}$ was noted. Laboratory food pellets were available ad libitum to the rats during the test. ${ }^{48}$

Sucrose-induced biphasic response to noxious stimulus. On the day of experiment, the rat received freshly prepared 20\% sucrose (Sucrose GR, Merck Ltd, Mumbai, India) solution for $4 \mathrm{~h}$. Fore paw lick latency (FPL), a phasic pain test, was determined before (session I) and after $0.5 \mathrm{~h}, 1 \mathrm{~h}, 2 \mathrm{~h}, 2.5 \mathrm{~h}, 3 \mathrm{~h}$ and $4 \mathrm{~h}$ (sessions II-VII, respectively) of sucrose ingestion. ${ }^{26}$ The quantity of sucrose ingested during the test was noted.

\section{Fore paw lick latency}

Paw sensitivity to thermal noxious stimulus was assessed using hot plate analgesia meter (Omnitech, Woodland Hills, CA, USA), which was maintained at $52 \pm 1{ }^{\circ} \mathrm{C}$ by an inbuilt thermostat. Simultaneous with placement of rat on the hot plate, the timer was started by the pedal switch. The latency to lick the fore-paw(s) got frozen on the monitor, which was recorded as FPL. The 'cutoff duration was pre-set at $30 \mathrm{~s}$ to avoid thermal injury to the plantar surface of the paw during the test. ${ }^{26}$

\section{Histology}

At the end of study, the rat was deeply anaesthetised and killed by transcardial perfusion with saline $(0.9 \%)$ followed by paraformaldehyde (4\%) in $0.1 \mathrm{M}$ PBS. Brain and spinal cord were isolated, post-fixed in the same paraformaldehyde solution at $4{ }^{\circ} \mathrm{C}$ for $6 \mathrm{~h}$ and cryoprotected in sucrose $(30 \%)$ at $4^{\circ} \mathrm{C}$ for $48-72 \mathrm{~h}$. Brain was cut coronally ( $6 \mu \mathrm{m}$ thickness for c-Fos immunohistochemistry in Sham, SCI and SCI+MF rats; $30 \mu \mathrm{m}$ thickness for cresyl violet (CV) staining in VMHL, SCI+VMHL and SCI+VMHL+MF rats) using a cryostat (Microm HM 550 , Thermoscietific, Kalamzoo, MI, USA). Sectioning of SC (30- $\mu$ m thick) was started from the dorsal side of spinal cord. The sections were collected on poly L-lysine-coated slides. Similar sagittal sections from different rats were collected by selecting specific number (for example, every 30th section) of SC section. The above-mentioned procedure for cutting and collection of SC sections was strictly adhered to so as to enable us to compare nearly similarity of SC areas was various intra- and inter-group rats. In addition, the similarities were again confirmed by specific microscopic features-for example, arrangements of grey and white matter, and so on.

Frozen SC sections were utilised for CV and luxol fast blue (LFB) staining to calculate the lesion volume and assess the degree of myelination, respectively.

\section{c-Fos immunohistochemistry}

Fos-immunoreactive (Fos-ir) profile in VMH was determined to assess its neuronal activity. After blocking endogenous peroxidase, the sections were treated with normal serum and then incubated overnight at $4{ }^{\circ} \mathrm{C}$ with the primary antibody (1:200, Santa Cruz Biotechnology, Santa Cruz, CA, USA). The sections were then incubated with a biotinylated rabbit secondary antibody for $1 \mathrm{~h}$ at room temperature. Bound secondary antibody was amplified during $30 \mathrm{~min}$ incubation of the sections in the avidin-biotin complex (Vectastain ABC Elite Kit, Vector Laboratories, Burlingame, CA, USA). Antibody complexes were visualised by immersing the tissues in $2 \%$ diaminobenzidine (Vector Laboratories). This reaction was stopped by rinsing the sections in $50 \mathrm{~mm}$ Tris-HCL (pH 7.4). Sections were then covered with a cover slip using mount quick (Daido Sangyo, Saitama, Japan). Three sections per rat ( $n=6$ in 
each group) were selected for the analysis. They were inspected through a microscope (Eclipse 80i, Nikon, Tokyo, Japan), and Fos-ir cells were counted by using software (NIS ELEMENTS, Version AR3, Nikon). Cells were considered c-Fos immunoreactive, if they contained dark, punctuate, nuclear immunostaining. ${ }^{49}$

\section{CV staining}

The sections were CV stained to identify and confirm the lesion of VMH and to calculate the lesion volume in SC. Briefly, after rehydration, the sections were treated with warm $0.1 \% \mathrm{CV}$ solution for 5-10 min. Later, the sections were rinsed with distilled water and dehydrated with two changes each for $5 \mathrm{~min}$ in $95 \%$ and $100 \%$ ethanol, respectively. The sections were cleared with Xylene, mounted with DPX, cover slipped and then visualised (Eclipse 80i, Nikon). The quantification of lesion volume was performed from every fifth section per spinal cord (six rats per group). A total number of 14-15 sections per rat was used for quantification.

\section{LFB staining}

SC tissue sections were stained with LFB to assess the myelination. Briefly, the frozen sections were warmed to room temperature, and they were rehydrated by dipping in distilled water for $10 \mathrm{~min}$. Hydrated tissue sections were then immersed in LFB stain and kept overnight in oven at $56^{\circ} \mathrm{C}$. Excess stain was rinsed off initially with $95 \%$ ethyl alcohol and then with distilled water. The sections were then differentiated by lithium carbonate solution for $30 \mathrm{~s}$ followed by $70 \%$ ethyl alcohol for another $30 \mathrm{~s}$. After rinsing in distilled water, the sections were checked microscopically to determine the clarity of grey matter and sharpness of white matter. Later the sections were counterstained in the Nuclear Fast Red (Vector laboratories, Cat no: H-3403) solution for 2-5 min and rinsed in distilled water for $5 \mathrm{~min}$. They were then dehydrated with two changes each for $5 \mathrm{~min}$ in 95\% ethanol and 100\% ethanol, respectively. Sections were cleared with xylene, mounted with DPX, cover slipped and then visualised (Eclipse 80i, Nikon). The volume of demyelination was calculated from every fifth section per spinal cord (six rats per group). A total number of 14-15 sections per rat was used for quantification. Spinal cord tissue staining deep blue was interpreted as intact white matter. ${ }^{50}$

\section{GAP-43 immunohistochemistry}

Spinal cord sections were incubated in blocking solution (PBS containing 10\% normal goat serum, $0.1 \%$ Triton $\mathrm{X}-100$ ) for $2 \mathrm{~h}$ at room temperature, followed by overnight incubation at $4{ }^{\circ} \mathrm{C}$ in a humidified chamber with rabbit anti-growth-associated protein-43 (GAP-43; 1:500; Sigma Aldrich, St Louis, MO, USA) to visualise neuronal sprouting, mouse anti-NeuN (1:1000; Abcam, Cambridge, UK) to visualise mature neurons and mouse anti-NF 160 (1:400; Sigma Aldrich) to axons. The antibodies were prediluted in PBS containing $0.5 \%$ normal goat serum and $0.1 \%$ Triton X-100. Fluorescent secondary antibodies were either alexa 488-conjugated goat anti-rabbit IgG (1:200; Abcam, Cambridge, MA, USA) or alexa 647-conjugated goat antimouse IgG (1:200; Abcam). Autofluorescence masking was performed by incubating the sections with $0.5 \% \mathrm{CuSO}_{4}$ in $50 \mathrm{mM}$ ammonium acetate buffer for $15 \mathrm{~min}$. After washing, sections were incubated with $0.1 \%$ DAPI (4', 6-diamidino-2-phenylindole dihydrochloride; Sigma Aldrich) for $10 \mathrm{~min}$ at room temperature for staining the nuclei. Sections were also double labelled with NeuN or NF 160 with GAP-43. Slides were cover slipped with the fluoroshield mounting medium (Sigma Aldrich), and images were obtained using a laser scanning confocal microscope (TCS SP2, Leica, Germany). Quantitative analysis of GAP- $43^{+}$cells was performed by a blinded observer using NIS-ELEMENT software. For cell density determination, the number of positively labelled cells was counted from 5 rostral and 5 caudal areas within $1 \mathrm{~mm}$ length of lesion epicentre at $\times 40$ magnification. Measurements were carried out over 10 images per section per rat ( $n=6$ in each group).

\section{Study plan}

The surgeries (Laminectomy, SCI and VMHL) were performed after obtaining the baseline data of all the parameters. SCI was performed immediately after $\mathrm{VMH}$ lesion on the same day in the SCI+VMHL group. FI and WI were measured daily and BWT was recorded weekly. Taste preference test (performed in 5 consecutive days for various tastants) was performed at week $1,3,5$ and 7 , whereas sucrose-induced response to noxious stimulus was performed at week 4 and 8 . SCI+MF and SCI+VMHL+MF group of rats were exposed to ELF-MF daily for $2 \mathrm{~h}$ from 1100 hours to 1300 hours. Rats were killed at the end of week 8 and their brain and spinal cord were isolated for histological analysis.

\section{Statement of ethics}

We certify that all applicable institutional and governmental regulations concerning the ethical use of animals were followed during the course of this research.

\section{Statistical analysis}

Statistical analysis was carried out using Stata 11.0 (StataCorp, College station, TX, USA). Data was presented as mean \pm S.D. or median (min-max) as appropriate. All the parameters were analysed among the groups over a period of time using generalised estimating equation as the observations were correlated. Data of sodium chloride and citric acid intake were analysed among the groups at each time point using the Kruskal-Wallis test and within groups by the Wilcoxon signed-rank test, as the data were not following normal distribution. For multiple comparisons, Bonferroni correction was carried out after generalised estimating equation and the Kruskal-Wallis test. $P$-value $<0.05$ was considered statistically significant.

\section{RESULTS}

\section{Feeding behaviour}

FI and CI. In comparison with Sham rats, the FI was significantly less in SCI rats, whereas more in VMHL rats during week 1 through 8 (Table 1). The FI in SCI+VMHL rats was significantly more vs SCI rats during the study period. However, no difference was observed between SCI+VMHL and VMHL rats, except during week 1 and 2.

The FI in SCI+MF rats was significantly more $(P<0.001)$ vs SCI rats during week 1 through 8 . However, no difference was observed between SCI+VMHL and SCI+VMHL+MF rats. The CI in various rat groups varied similarly to FI.

Water intake. During week 1 through 8 , the WI in SCI rats was significantly less, whereas it significantly increased in VMHL rats when compared with Sham rats (Table 2). However, the WI was more in SCI+VMHL rats vs SCI rats. No difference was observed between SCI + VMHL and VMHL rats, except during week 1.

The WI in SCI+MF rats was significantly more $(P<0.001)$ vs SCI rats during week 1 through 8 . However, no difference was observed between SCI+VMHL and SCI+VMHL+MF rats during the study period.

Body weight. The BWT remained significantly less in SCI rats, whereas it increased significantly in VMHL rats vs Sham rats during week 1 through 8 . The BWT in SCI+VMHL rats was significantly more vs SCI and less vs VMHL rats during week 1 through 8 .

The BWT in SCI+MF rats increased significantly vs SCI rats during week 1 through 8 . No significant difference in BWT was observed between SCI+VMHL and SCI+VMHL+MF rats (Table 3).

\section{Taste preference test}

Sodium chloride $(\mathrm{NaCl})$. $\mathrm{NaCl}$ intake was more in SCI rats during week 3-7, whereas less in VMHL rats during week 5-7 vs Sham rats. However, $\mathrm{NaCl}$ intake in VMHL and SCI+VMHL rats was comparable (Table 4). $\mathrm{NaCl}$ intake decreased in SCI+MF rats vs SCI rats and was comparable to Sham rats during week 5-7. No statistical significant difference was observed between SCI+VMHL and SCI+VMHL $+\mathrm{MF}$ rats. 


Sucrose. The sucrose intake in SCI rats was significantly less vs Sham rats during week 3-7. In the VMHL and SCI+VMHL rats, the sucrose intake was significantly more vs Sham and SCI rats, respectively, during week 1 through 7 (Table 4).

Sucrose intake in SCI+MF rats significantly increased vs SCI rats during week 3-7 and was comparable to Sham rats. No statistical significant difference was observed between SCI+VMHL and SCI + VMHL+MF rats.

Saccharin. In comparison with Sham rats, intake for saccharin was less in SCI rats, whereas it increased in VMHL rats from week 1 to 7. There was no difference between SCI+VMHL rats and VMHL rats. In comparison with SCI rats, SCI+VMHL rats showed greater saccharin intake from week 1 through 7 (Table 4).

SCI+MF rats showed higher intake of saccharin vs SCI rats, whereas it was comparable to Sham rats during week 5-7. No statistical significant difference was observed between SCI+VMHL and SCI $+\mathrm{VMHL}+\mathrm{MF}$ rats.

Citric acid. In comparison with Sham rats, citric acid intake was more in SCI rats during week 1 through 7, and it was less in VMHL rats during week 3-7. Citric acid intake in VMHL and SCI+VMHL rats was comparable (Table 4).

SCI+MF rats showed lower intake of citric acid when compared with SCI rats, whereas it was comparable to Sham rats during week 1 through 7. No statistical significant difference was observed between $\mathrm{SCI}+\mathrm{VMHL}$ and SCI+VMHL+MF rats.

Quinine hydrochloride. None of the groups preferred to ingest quinine hydrochloride solution at any study point.

\section{Sucrose-induced biphasic response to noxious stimulus}

Week 0 . In all groups, FPL increased at $0.5 \mathrm{~h}$ followed by reversal to baseline FPL at 1,2 and $2.5 \mathrm{~h}$ and later decreased at 3 and $4 \mathrm{~h}$ after sucrose ingestion (Figure 1a)

Week4. At week 4, baseline FPL was decreased in all rat groups, except Sham rats. In Sham rats, FPL responses to sucrose ingestion were similar to that at week 0. However, in SCI rats, FPL increased only at alternate study points $(0.5,2$ and $3 \mathrm{~h})$ vs their baseline FPL. SCI+MF rats showed higher FPL at $0.5,1$ and $2 \mathrm{~h}$ followed by reversal to baseline value at 2.5 and $3 \mathrm{~h}$, whereas lower FPL at $4 \mathrm{~h}$ after sucrose ingestion. The FPL did not vary significantly in VMHL, SCI+VMHL and SCI+VMHL+MF rats from their baseline value at any study time points (Figure 1b).

Week8. At week 8, the FPL of Sham rats was comparable to week 0 and week 4. In SCI rats, FPL was higher at $0.5,1,2,2.5$ and $3 \mathrm{~h}$ but was comparable at $4 \mathrm{~h}$ vs baseline FPL. Sham and SCI+MF rats showed similar responses in FPL to sucrose ingestion-that is, initial increase at $0.5 \mathrm{~h}$, reversal to baseline FPL at 1,2 and 2.5 and decrease at 3 and $4 \mathrm{~h}$. The FPL did not vary significantly in VMHL, SCI + VMHL and SCI+VMHL+MF rats from baseline value at any other study time points (Figure 1c).

\section{c-Fos immunohistochemistry}

Robust c-Fos expression in VMH of SCI rats (141.53 \pm 42.49 ; $P \leq 0.001)$ was observed when compared with Sham rats $(7 \pm 4.58)$ at week 8 . They were specifically seen at dorsomedial division of VMH $(\mathrm{dm}-\mathrm{VMH})$. In SCI+MF rats, c-Fos expression decreased $(26.42 \pm 14.7 ; P \leq 0.001)$ vs SCI rats (Figure 2$)$. 


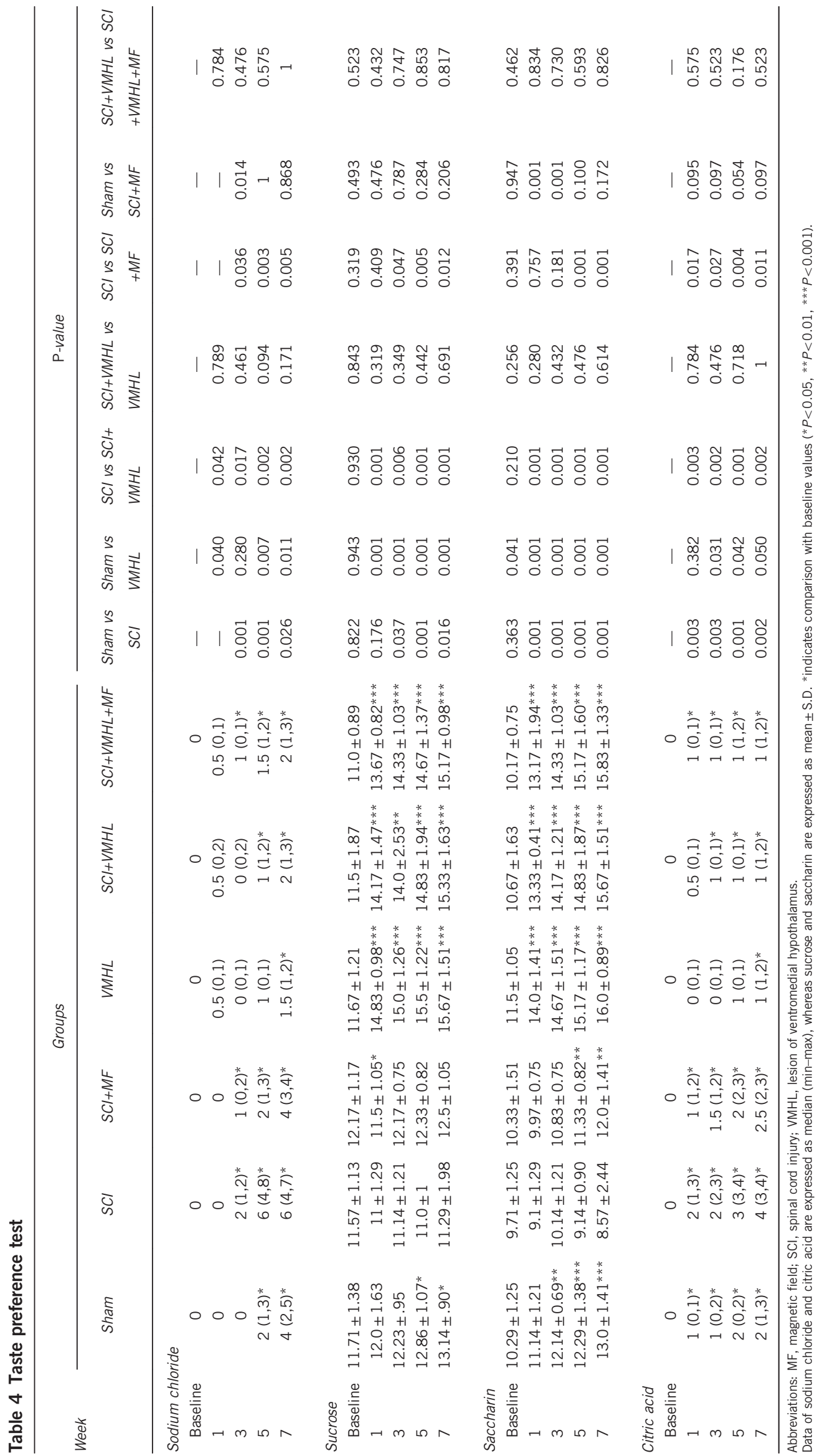




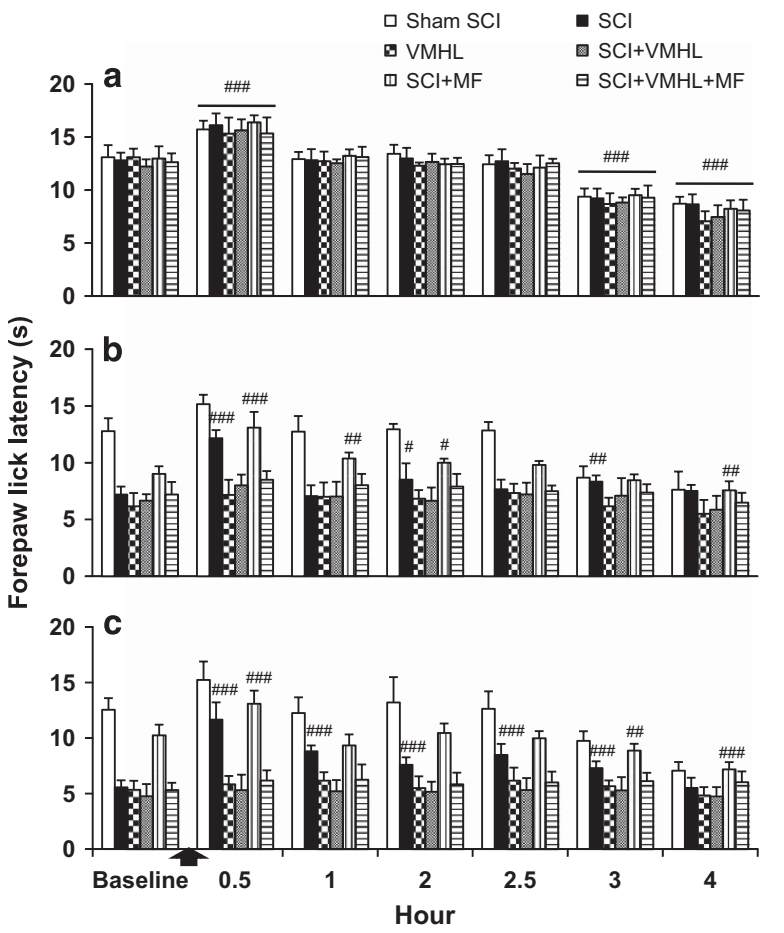

d

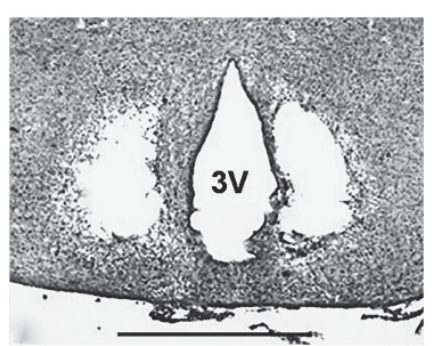

Figure 1 Nociceptive responses to sucrose ingestion (a) Week 0 , (b) week 4 and (c) week 8. (d) Brain section with bilateral electrolytic lesion of $\mathrm{VMH}$. $3 \mathrm{~V}$, third ventricle. Scale bar, $1000 \mu \mathrm{m}$. Arrow mark indicates sucrose ingestion. \#indicates comparison with baseline value.

\section{CV staining}

In all VMH lesioned rats (VMHL, SCI+VMHL and SCI+VMHL $+\mathrm{MF}$ ), CV staining of brain showed bilateral tissue loss in the $\mathrm{VMH}$, thereby confirming the lesion of $\mathrm{VMH}$ (Figure 1d).

CV staining of SC sections of every rat in all the groups revealed bilateral tissue loss from the lesion epicentre. It was more in spinal cord sections of SCI rats as compared with SCI+MF rats (Figures 3a and $\mathrm{b}$ ). The total volume of tissue damage in the SCI group was $17.95 \pm 4.89 \mathrm{~mm}^{3}$, whereas in the SCI+MF group it decreased significantly to $13.36 \pm 4.12 \mathrm{~mm}^{3}$, indicating attenuation in the extent of damage.

\section{LFB staining}

LFB staining of spinal cord revealed reduced volume $\left(15.43 \pm 4.42 \mathrm{~mm}^{3}\right)$ of demyelination in the lesion area of SCI+MF rats compared with SCI rats $\left(20.52 \pm 5.26 \mathrm{~mm}^{3}\right.$; Figures $3 \mathrm{c}$ and d).

\section{GAP-43 immunohistochemistry}

GAP-43 immunoreactivity in the spinal cord sections was utilised to study the neuronal sprouting. Negligible GAP-43 expression was seen at the lesion border of SC sections of all the SCI rats (Figure 4a), whereas in SCI+MF rats their expression was significantly greater (Figure 4g). Co-localisation of GAP-43 with NeuN and NF 160 in SCI $+\mathrm{MF}$ rats suggests that mature and viable neurons are regenerating axons (Figure 5).

\section{DISCUSSION}

The present study was aimed to investigate the probable involvement of $\mathrm{VMH}$ in the aberrant feeding behaviour of spinalised rats and the influence of MF exposure on it. The data indicate hypophagia, hypodipsia, loss of BWT, increased preference for salt and sour tastants and sucrose-induced prolonged analgesia to nociceptive stimulus after complete transection of SCI in rats. These behavioural responses in spinalised rats are indicative of the involvement of $\mathrm{VMH}$, which was supported by greater c-Fos immunoreactivity in VMH. Greater c-Fos immunoreactivity clearly supports its stimulated status after SCI. To confirm and decipher the precise involvement of $\mathrm{VMH}$, its lesion was performed in SCI rats, which resulted in attenuation of SCI effects.

Daily exposure of SCI rats to MF significantly restored the above-mentioned effects of SCI. MF exposure resulted in reduced lesion volume, increased myelination and signs of regeneration at the site of SCI. The present study suggests that complete spinal cord transection in rats leads to stimulation of $\mathrm{VMH}$, which reflects in its control on feeding behaviour. However, exposure to MF results in partial restoration of VMH functions probably secondary to limitation of the injury processes in the spinal cord.

The cumulative intake of food and water was decreased in spinalised rats immediately after surgery, which is in parallel with our previous report in a comparable rat model of SCI. ${ }^{7}$ SCI-induced reduction in feeding behaviour is reported in rats by other researchers too, albeit in different injury modes and levels. ${ }^{51-53}$ SCI rats exhibit reduction in oesophageal-gastric relaxation reflex, GI motility, ${ }^{53}$ gastric emptying ${ }^{54}$ and GI transit that results from gastro-inhibitory signals. ${ }^{9-11,55}$ Dye recovery studies have shown reduced transit time in jejunum $(64.1 \%)$ and ileum $(73.6 \%)$ of SCI rats. These effects are attributed to permanent changes in GI tract and homoeostatic mechanisms. ${ }^{51}$ We suspect that such global changes in GI movements are unlikely to be the only peripheral deficit but possibly are the effects of supraspinal structures.

The supraspinal changes in sensorimotor cortex, thalamus and brain stem have been well documented in the literature by using elegant state-of-art techniques. Morrow et al. ${ }^{56}$ have reported increase in blood flow to arcuate nucleus of SCI rats. The arcuate nucleus has rich monosynaptic connections with $\mathrm{VMH},{ }^{57}$ which again indicates involvement of medial hypothalamus after SCI.

The FI, WI, BWT and taste preference are the functions of VMH. Although lesion of VMH causes hyperphagia, hyperdipsia, obesity and preference for high lipid and carbohydrate diet, its stimulation causes the opposite effects. VMH stimulation is also associated with inhibition of gastric motility, ${ }^{58}$ which is also observed in SCI rats. In addition to alteration in feeding behaviour, our SCI rats exhibited higher preference for salt $(\mathrm{NaCl})$ and sour (citric acid) tastants, whereas lower preference for sweet (sucrose and saccharin) tastants. The above data in conjunction with the literature further support the probable involvement and stimulation of $\mathrm{VMH}$, leading to alteration in feeding behaviour of spinalised rats.

$\mathrm{VMH}$ also exerts a fine, complex and dynamic control on the biphasic pattern of pain response (that is, initial analgesia at $30 \mathrm{~min}$ followed by eualgesia at $1 \mathrm{~h}$ and hyperalgesia at $3 \mathrm{~h}$ ) when the palatable food is ingested. ${ }^{26}$ Either electrolytic lesion or 2-deoxy-D- 

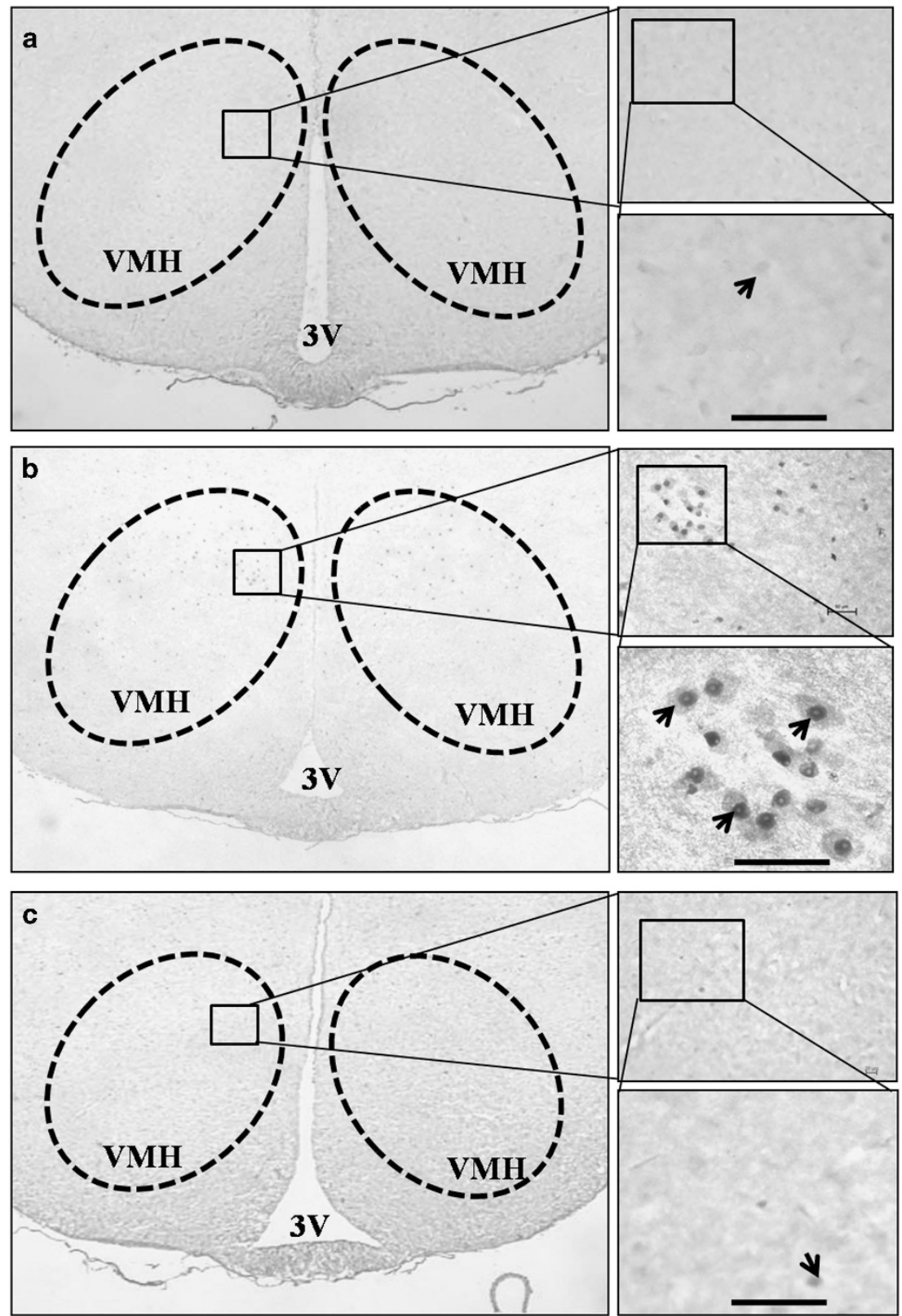

Figure 2 Representative photomicrographs showing c-Fos expression in $\mathrm{VMH}$ of Sham (a), $\mathrm{SCl}$ (b) and $\mathrm{SCl}+\mathrm{MF}$ (c) rats. Number of c-Fos ${ }^{+}$neurons was significantly less in $\mathrm{SCl}+\mathrm{MF}$ rats (c) as compared with $\mathrm{SCl}$ rats (b). Scale bar, $50 \mu \mathrm{m}$.

glucose (2DG) micro infusion in VMH attenuated sucrose-fed hyperalgesia, ${ }^{22,25}$ indicating a critical involvement of $\mathrm{VMH}$ in the mediation of biphasic pain response after ad libitum sucrose ingestion. $\mathrm{VMH}$ contains a large number of glucoresponsive neurons ${ }^{28,59}$ that possess the ability to exert influence on satiety, palatability and palatability-induced modulation of pain. In our SCI rats, there was a significant prolongation in the initial analgesic response $(0.5-3 \mathrm{~h})$ after sucrose ingestion, over and above baseline hyperalgesia. These data further support involvement of $\mathrm{VMH}$.

The nature of involvement of $\mathrm{VMH}$, that is, stimulation/ inactivation, was studied by c-Fos immunohistochemistry. c-Fos protein represents the product of the immediate-early gene, which has been used as a marker of neuronal activity. ${ }^{60}$ The results indicate significant expression of c-Fos in the dorsomedial division of $\mathrm{VMH}$
$(\mathrm{dm}-\mathrm{VMH})$ in the spinalised rats, suggesting its activated status. The $\mathrm{dm}-\mathrm{VMH}$ is particularly dense with neurons that respond to feeding cues, whereas the capsular region is critically important for receiving and probably processing afferent informations. ${ }^{61}$

To further confirm the involvement of $\mathrm{VMH}$, its lesion was performed immediately after SCI. VMH lesion in spinalised rats attenuated the effects of SCI on feeding-related parametersnamely, the FI, WI, taste preference and sucrose-induced biphasic pain, which were found to be comparable to VMHL rats. Therefore, the data obtained from spinalised rats with VMH lesion support the involvement of VMH after SCI in terms of its stimulation. Needless to say that, the involvement of $\mathrm{VMH}$ adds to the list of various other parts of the brain that are affected consequent to spinalisation. 


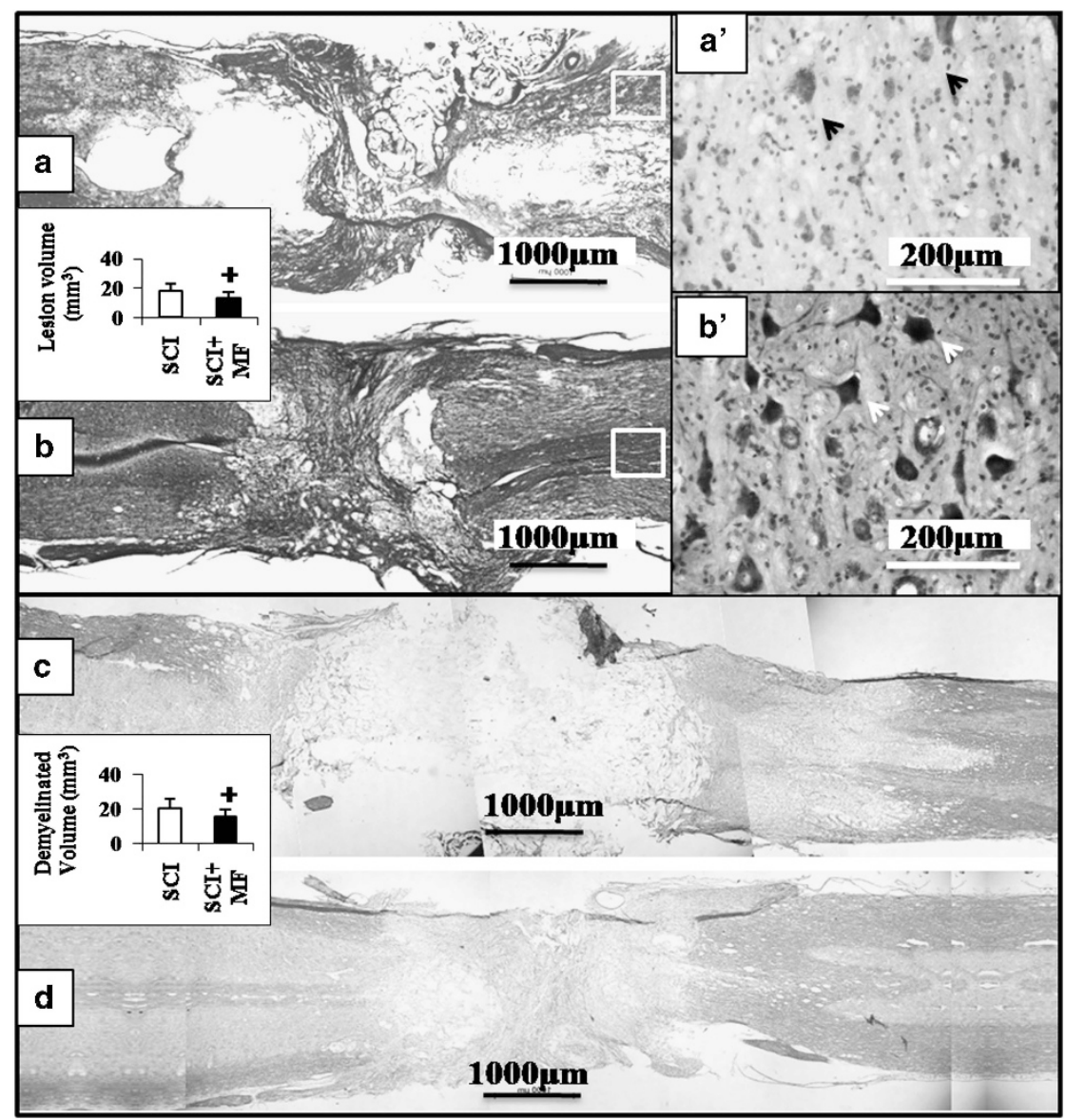

Figure 3 Photomicrograph of CV and LFB-stained spinal cord sections. Tissue degeneration at the lesioned area of spinal cord was more in SCl rats (a) than $\mathrm{SCl}+\mathrm{MF}$ (b) rats. In SCl+MF rats, viable motor neurons were indicated by arrowheads (b'), whereas in $\mathrm{SCl}$ rats (a') the viable motor neurons were absent and dense inflammatory cells were observed. (c, d) LFB-stained spinal cord sections of SCl and $\mathrm{SCl}+\mathrm{MF}$ rats, respectively. Blue-stained region shows the myelinated area (white matter). Demyelinated volume in SC sections of MF-exposed rats was less than that of SCl rats. ${ }^{+}$sign in the inset graph indicates comparison with $\mathrm{SCl}$ rats. A full color version of this figure is available at the Spinal Cord journal online.

The BWT was significantly decreased in our SCI rats- that is in line with the existing literature. ${ }^{51}$ Loss of BWT can be attributed to reduction in food and WI besides marked osteoporosis, ${ }^{44,62,63}$ sublesional disuse wasting of muscles ${ }^{64,65}$ and other metabolic effects ${ }^{66}$ in transection model of SCI. Primeaux et al. ${ }^{51}$ reported a decrease in BWT after complete transection of spinal cord at T3 in female rats, which was maintained until week 18. Despite an increase in mean energy intake during week 16-18, the authors suggested that the decrease in BWT in SCI rats was not due to hypophagia but possibly due to permanent changes in homoeostatic mechanismsnamely, atrophy of muscles, GI transit and absorption. They concluded that regulatory mechanisms for FI and BWT are dissociated. However, our study does not agree with their study because our rats were hypophagic and the food and WI were measured from day 1 after surgery. In contrast, Primeaux et al. ${ }^{51}$ have measured the mean energy intake during week 16-18 only.

MF exposure to SCI rats resulted in significant improvement of their FI, WI and BWT during week 1 through 8, albeit less than the Sham rats indicating partial recovery. This is further supported by reduction in c-Fos immunoreactivity of VMH. However, when MF was provided to $\mathrm{VMH}$-compromised SCI rats, there was no further improvement in the data obtained from VMH-compromised SCI rats. These observations lead us to logically conclude that the daily exposure of SCI rats to MF resulted in the reduction of stimulation of VMH.
MF exposure to SCI rats restored the taste preferences and the biphasic pattern of sucrose-induced pain modulation. However, the MF exposure to VMH-compromised SCI rats did not restore the biphasic pattern, as the effect of VMH lesion predominated in them. These data again revealed the beneficial effect of MF on VMH function.

To study the influence of MF exposure on SCI-induced spinal segmental changes, CV staining of SC was performed. Remarkable reduction in the lesion volume was noticed in MF-exposed SCI rats, which is supported and contributed by the presence of greater myelination around the lesion site as revealed by LFB staining. Moreover, significant increase in the GAP-43, which is an endogenous indicator of axonal regeneration, and its co-localisation with NeuN (mature neuronal marker) and NF 160 (marker of axon) in the MF-exposed SCI rats indicates the neuronal sprouting and regeneration that contributes to reduction in lesion volume.

Successful regeneration in CNS neurons has been closely correlated with GAP-43 expression. ${ }^{67}$ GAP-43 generally gets concentrated at the axonal growth cone, where it seems to have an important role in the transduction of growth cone guidance signals. Some degree of functional recovery, however, can be observed without management. ${ }^{68}$ Our SCI rats also exhibited minimal GAP-43 expression around the lesion site without MF exposure. 


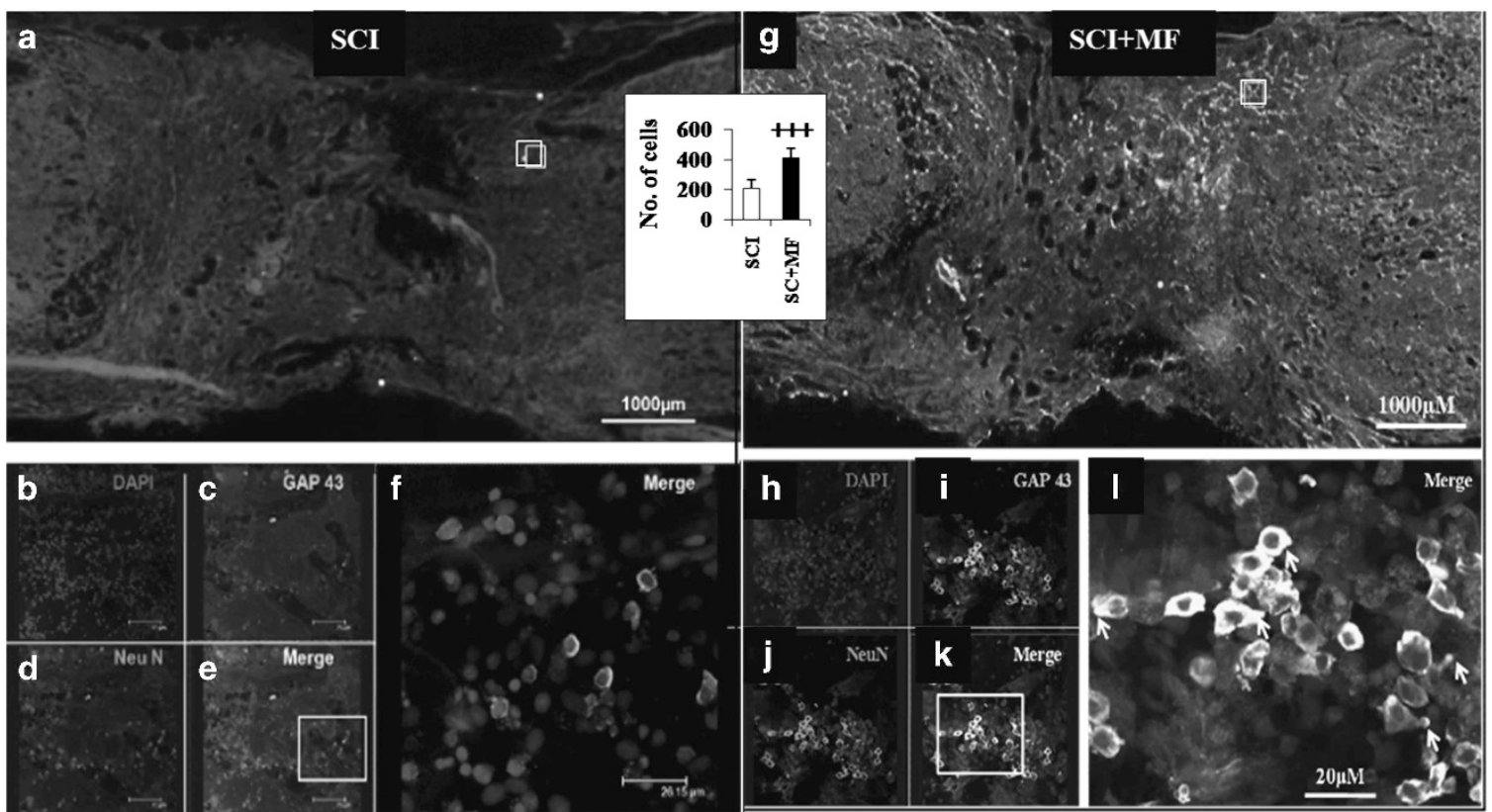

Figure 4 Effect of MF exposure on GAP-43 expression in spinalised rats: Negligible GAP-43 immunoreactivity was evident in spinal cord sections of SCI (a) rats. (b-e) are showing DAPI, GAP-43, NeuN staining and merged picture, respectively. (f) Enlarged picture from e. MF-exposed SCl rats (g) showed significant GAP-43 immunoreactivity at the lesion border. (h-k) are showing DAPI, GAP-43, NeuN staining and merged picture, respectively. (I) Enlarged picture from k. Co-localisation of GAP-43 and NeuN indicates neuronal sprouting (arrow marks). ${ }^{+}$sign in the inset graph indicates comparison with $\mathrm{SCl}$ rats. A full color version of this figure is available at the Spinal Cord journal online.
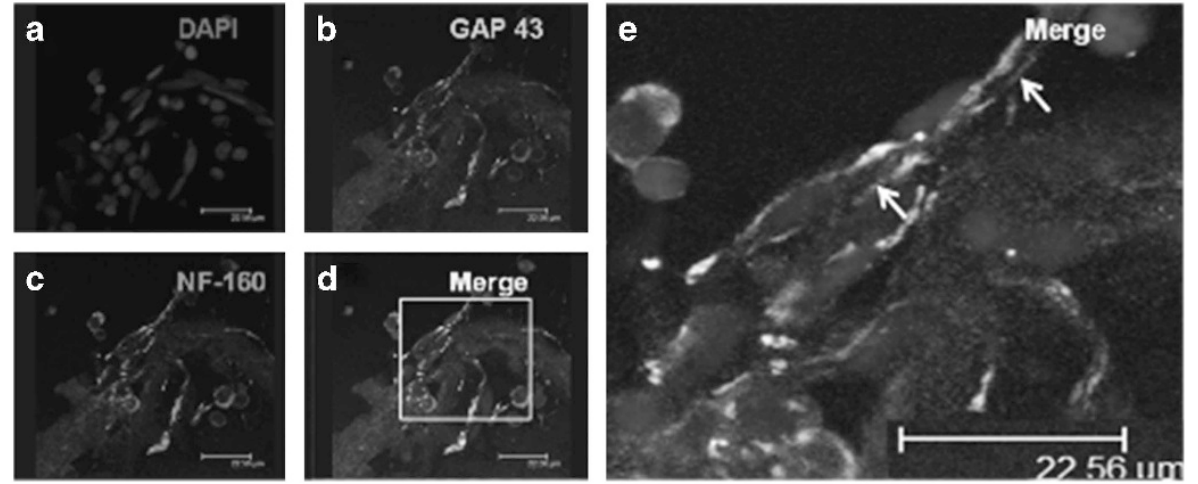

Figure 5 Photomicrograph showing axonal sprouting in MF-exposed SCl rats. (a-d) are showing DAPI, GAP-43, NF 160 staining and merged picture, respectively. (e) Enlarged picture from d. Co-localisation of GAP-43 and NF 160 expression indicates axonal sprouting (arrow marks) in SCl+MF rats. A full color version of this figure is available at the Spinal Cord journal online.

Although the exact mechanism for these beneficial effects of MF is not known, several possibilities have been proposed. ELF-MF stimulation creates intense rapid electric fields that can penetrate soft tissue and bone to reach deep structures including the nervous system. If the created electric field is of appropriate intensity, amplitude and duration, the neural microenvironment can be altered besides being stimulated through depolarisation. ${ }^{69}$ This may help in the maintenance of functional integrity of the neurons after injury. In addition, the restoration of the neurochemicals (for example: brain-derived neurotrophic factor, glial cell-derived neurotrophic factor) complements and provides functional conducive microenvironment for perpetuation of further regeneration. It is also found to promote osteogenesis, ${ }^{70}$ nerve regeneration; ${ }^{42}$ besides reducing apoptosis, ${ }^{71}$ oxidative stress $^{72}$ and inflammation. ${ }^{73}$
This study reports the beneficial effect of MF on SCI-induced energy imbalance in rats, which can be attributed to the change in $\mathrm{VMH}$ function. This change in the $\mathrm{VMH}$ function is possibly secondary to spinal changes-namely, reduction in degeneration, reduction in lesion volume and enhancement in neuronal regeneration around the injury site. This is the first report to suggest a non invasive, safe, non pharmacological management strategy that limits the sequelae of SCI.

Further studies in this area are needed to carry forward the research outcome of this study for the benefit of SCI patients. ${ }^{31,47}$

\section{DATA ARCHIVING}

There were no data to deposit. 


\section{CONFLICT OF INTEREST}

The authors declare no conflict of interest.

\section{ACKNOWLEDGEMENTS}

This work was supported by Indian Council of Medical Research (ICMR), New Delhi, India. We gratefully acknowledge Mr Purushottam Samal and Mr Sanjeev for the technical help and Mrs Mamta Sharma for the secretarial assistance.

1 Wong S, Graham A, Green D, Hirani SP, Forbes A. Nutritional supplement usage in patients admitted to a spinal cord injury center. J Spinal Cord Med 2013; 36: 645-651.

2 Schwab ME, Bartholdi D. Degeneration and regeneration of axons in the lesioned spinal cord. Physiol Rev 1996; 76: 319-370.

3 Hulsebosch CE. Recent advances in pathophysiology and treatment of spinal cord injury. Adv Physiol Educ 2002; 26: 238-255.

4 Dietz V, Curt A. Neurological aspects of spinal-cord repair: promises and challenges. Lancet Neurol 2006; 5: 688-694.

5 Wang R, Hong J, Lu M, Neil JE, Vitek MP, Liu X et al. Apo E mimetic ameliorates motor deficit and tissue damage in rat spinal cord injury. J Neurosci Res 2014; 92: 884-892.

6 Wang W, Wang F, Liu J, Zhao W, Zhao Q, He M et al. SNAP25 ameliorates sensory deficit in rats with spinal cord transection. Mol Neurobiol 2014; 50: 290-304.

7 Kumar S, Jain S, Behari J, Avlev VD, Mathur R. Effect of magnetic field on food and water intake and body weight of spinal cord injured rats. Indian J Exp Biol 2010; 48: 982-986.

8 Groah SL, Mark SN, Inger HL, Alexander L, Larry FH, Emily W et al. Nutrient intake and body habitus after spinal cord injury: an analysis by sex and level of injury. J Spinal Cord Med 2008; 32: 25-33.

9 Holmes GM. Upper gastrointestinal dysmotility after spinal cord injury: is diminished vagal sensory processing one culprit? Front Physiol 2012; 3: 277.

10 Gondim FA, de Alencar HM, Rodrigues M, da Graca L, dos Santos R, Rola FH. Complete cervical or thoracic spinal cord transections delay gastric emptying and gastrointestinal transit of liquid in awake rats. Spinal Cord 1999; 37: 793-799.

11 Gondim FA, Rodrigues CL, da Graca JR, Camurca FD, de Alencar HM, dosSantos AA et al. Neural mechanisms involved in the delay of gastric emptying and gastrointestinal transit of liquid after thoracic spinal cord transection in awake rats. Auton Neurosci 2001; 87: 52-58.

12 Hetherington AW, Ranson SW. The spontaneous activity and food intake of rats with hypothalamic lesions. Am J Physiol 1942; 136: 609-617.

13 Anand BK, Brobeck JR. Localization of a "feeding center" in the hypothalamus of the rat. Proc Soc Exp Biol Med 1951; 77: 323-324.

14 Oomura Y, Kimura K, Ooyama H, Maeo T, Iki M, Kuniyoshi N. Reciprocal activities of ventromedial and lateral hypothalamic areas of cats. Science 1964; 143: 484-485.

15 King BM. The rise, fall, and resurrection of the ventromedial hypothalamus in the regulation of feeding behaviour and body weight. Physiol Behav 2006; 87: 221-244.

16 Teitelbaum P. Sensory control of hypothalamic hyperphagia. J Comp Physiol Psychol 1955; 48: 156-163.

17 Levison MJ, Frommer GP, Vance WB. Palatability and caloric density as determinants of food intake in hyperphagic and normal rats. Physiol Behav 1973; 10: 455-462.

18 Peters RH, Mark WG. Finickiness in VMH rats also results from the lesions, not just from obesity. Physiol Psychol 1980; 8: 93-96.

19 Weingarten HP. Diet palatability modulates sham feeding in $\mathrm{VMH}$-lesion and normal rats: implications for finickiness and evaluation of sham-feeding data. J Comp Physiol Psychol 1982; 96: 223-233.

20 Bray GA, Inoue S, Nishizawa Y. Hypothalamic obesity. The autonomic hypothesis and the lateral hypothalamus. Diabetologia 1981; 20: 366-377.

21 Bray GA. Hypothalamic and genetic obesity: an appraisal of the autonomic hypothesis and the endocrine hypothesis. Int J Obes 1984; 8: 119-137.

22 Mukherjee K, Mathur R, Nayar U. Effect of VMH lesion of sucrose fed nociceptive responses. Jpn J Physiol 2000; 50: 395-404.

23 Mukherjee K, Mathur R, Nayar U. Ventromedial hypothalamic mediation of sucrose feeding induced pain modulation. Pharmacol Biochem Behav 2001; 68: 43-48.

24 Mukherjee K, Mathur R, Nayar U. Hyperalgesic response in rats fed sucrose from weaning to adulthood: role of VMH. Pharmacol Biochem Behav 2002; 73: 601-610.

25 Dutta R, Mukherjee K, Mathur R. Effect of VMH lesion on sucrose fed analgesia in formalin pain. Jpn J Physiol 2001; 51: 63-69.

26 Suri M, Jain S, Mathur R. Pattern of biphasic response to various noxious stimuli in rats ingesting sucrose ad-libitum. Physiol Behav 2010; 101: 224-231.

27 Oomura YH, Ooyama M, Yamamoto T, Faka F. Reciprocal relationship of the lateral and ventromedial hypothalamus in the regulation of food intake. Physiol Behav 1967; 2: 97-115.

28 Ono T, Nishino H, Fukuda M, Sasaki K, Muramoto KI, Oomura Y. Glucoresponsive neurons in rat ventromedial hypothalamic tissue slices in vitro. Brain Res 1982; 232: 494-499.

29 Kumar S, Jain S, Velpandian T, Petrovich GY, D Avelev V, Behari J et al. Exposure to extremely low-frequency magnetic field restores spinal cord injury-induced tonic pain and its related neurotransmitter concentration in the brain. Electromagn Biol Med 2013; 32: 471-483.

30 Tecott LH. Serotonin and the orchestration of energy balance. Cell Metab 2007; 6: 352-361.

31 Haleem DJ. Exaggerated feedback control decreases brain serotonin concentration and elicits hyperactivity in a rat model of diet-restriction-induced anorexia nervosa. Appetite 2009; 52: 44-50.

32 Cho H, Choi YK, Lee DH, Park HJ, Seo YK, Jung $H$ et al. Effects of magnetic nanoparticle-incorporated human bone marrow-derived mesenchymal stem cells exposed to pulsed electromagnetic fields on injured rat spinal cord. Biotechnol Appl Biochem 2013; 60: 596-602.

33 Crowe MJ, Sun ZP, Battocletti JH, Macias MY, Pintar FA, Maiman DJ. Exposure to pulsed magnetic fields enhances motor recovery in cats after spinal cord injury. Spine 2003; 28: 2660-2666.

34 Ahmed Z, Wieraszko A. Combined effects of acrobatic exercise and magnetic stimulation on the functional recovery after spinal cord lesions. J Neurotrauma 2008; 25: 1257-1269.

35 Ahmed Z, Wagdy M, Benjamin M, Mohamed S, Mohamed H, Ahmed S et al. Therapeutic effects of acrobatic exercise and magnetic field exposure on functional recovery after spinal cord injury in mice. Bioelectromag 2011; 32: 49-57.

36 Lin VW, Hsiao I, Kingery WS. High intensity magnetic stimulation over the lumbosacral spine evokes antinociception in rats. Clin Neurophysiol 2002; 113: 1006-1012.

37 Tsai PY, Wang CP, Chiu FY, Tsai YA, Chang YC, Chuang TY. Efficacy of functional magnetic stimulation in neurogenic bowel dysfunction after spinal cord injury. J Rehabil Med 2009; 41: 41-47.

38 Lin VW, Wolfe V, Perkash I. Micturition by functional magnetic stimulation. J Spinal Cord Med 1997; 20: 218

39 Lin VW, Hsiao I, Xu H, Bushnik T, Perkash I. Functional magnetic stimulation facilitates gastrointestinal transit of liquids in rats. Muscle Nerve 2000; 23: 919.

40 Lin VW, Nino-Murcia M, Frost F, Wolfe V, Hsiao I, Perkash I. Functional magnetic stimulation of the colon in persons with spinal cord injury. Arch Phys Med Rehabil 2001; 82: 167 .

41 Macias MY, Battocletti JH, Sutton $\mathrm{CH}$, Pintar FA, Maiman DJ. Directed and enhanced neurite growth with pulsed magnetic field stimulation. Bioelectromagnetics 2000; 21 : 272-286.

42 Mert T, Gunay I, Gocmen C, Kaya M, Polat S. Regenerative effects of pulsed magnetic field on injured peripheral nerves. Altern Ther Health Med 2006; 12: 42-49.

43 Das S, Kumar S, Jain S, Avelev VD, Mathur R. Exposure to ELF- magnetic field promotes restoration of sensori-motor functions in adult rats with hemisection of thoracic spinal cord. Electromagn Biol Med 2012; 31: 180-194.

44 Manjhi J, Kumar S, Behari J, Mathur R. Effect of extremely low frequency magnetic field in prevention of spinal cord injury-induced osteoporosis. J Rehabil Res Dev 2013; 50: $17-30$.

45 Ichiyama RM, Gerasimenko YP, Zhong H, Roy RR, Edgerton VR. Hindlimb stepping movements in complete spinal rats induced by epidural spinal cord stimulation. Neurosci Lett 2005; 383: 339-344.

46 Paxinos G, Watson C. The Rat Brain. In Stereotaxic Coordinates, 6th edn. Academic press: London, UK. 2009

47 Kirschvink JL Uniform magnetic fields and double wrapped coil systems: Improved techniques for the design of bioelectromagnetic experiments. Bioelectromagnetics 1992; 13: 401-411.

48 Brenda KS, Barker K, Schork MA, Kluger MJ. Development of altered taste preferences in tumor bearing rats. Appetite 1994; 23: 219-230.

$49 \mathrm{Chi} \mathrm{JH}$, Narita K, Ichimaru T, Murata T. Estrogen increases c-Fos expression in the paraventricular nucleus along with its anorexic effect in developing rats. J Reprod Dev 2011; 57: 365-372.

50 Ek CJ, Habgood MD, Dennis R, Dziegielewska KM, Mallard C, Wheaton B et al. Pathological changes in the white matter after spinal contusion injury in the rat. PLOS ONE 2012; 7: e43484.

51 Primeaux SD, Melissa T, Holmes GM. Effects of chronic spinal cord injury on body weight and body composition in rats fed a standard chow diet. Am J Physiol Regul Integr Comp Physiol 2007; 293: 1102-1109.

52 Jung J, Kim J, Hong SK, Yoon YW. Long-term follow-up of cutaneous hypersensitivity in rats with a spinal cord contusion. Korean J Physiol Pharmacol 2008; 12: 299-306.

53 Tong M. Holmes GM. Gastric dysreflexia after acute experimental spinal cord injury in rats. Neurogastroenterol Motil 2009; 21: 197-206.

54 Gondim FA, deAlencar HM, Rodrigues M, daGraca L, dos Santos R, Rola FH. Complete cervical or thoracic spinal cord transections delay gastric emptying and gastrointestinal transit of liquid in awake rats. Spinal Cord 1999; 37: 793-799.

55 Kabatas S, Yu D, He XD, Thatte HS, Benedict D, Hepgul KT et al. Neural and anatomical abnormalities of the gastrointestinal system resulting from contusion spinal cord injury. Neuroscience 2008; 154: 1627-1638.

56 Morrow TJ, Paulson PE, Brewer KL, Yezierski RP, Casey KL. Chronic, selective forebrain responses to excitotoxic dorsal horn injury. Exp Neurol 2000; 161: 220-226.

57 Verberne AJ, Sabetghadam A, Korim WS. Neural pathways that control the glucose counter regulatory response. Front Neurosci 2014; 26: 38.

58 Sharma KN, Anand BK, Dua S, Singh B. Role of stomach in regulation of activities of hypothalamic feeding centers. Am J Physiol 1961; 201: 593-598.

59 Oomura Y, Ono T, Ooyama J, Wayner MJ. Glucose and osmo sensitive neurons of the rat hypothalamus. Nature 1969; 222: 282-284.

60 Qiu MH, Chen MC, Huang ZL, Lu J. Neuronal activity (c-Fos) delineating interactions of the cerebral cortex and basal ganglia. Front Neuroanat 2014: 8: 13 . 
61 Saper CB, Swanson LW, Cowan WM. The efferent connections of the ventromedial nucleus of the hypothalamus of the rat. J Comp Neurol 1976; 169: 409-442.

62 Jiang SD, Jiang LS, Dia LY. Mechanisms of osteoporosis in spinal cord injury. Clin Endocrinol 2006; 65: 555-565.

63 Nassaralla C, Lyles KW. Possible way to reduce fracture rates in patients with traumatic spinal cord injury? Arch Phys Med Rehabil 2014; 95: 1021-1022.

64 Ohnishi Y, Iwatsuki K, Shinzawa K, Nakai Y, Ishihara M, Yoshimine T. Disuse muscle atrophy exacerbates motor neuronal degeneration caudal to the site of spinal cord injury. Neuroreport 2012; 23: 157-161.

65 Bodine SC. Disuse-induced muscle wasting. Int J Biochem Cell Biol 2013; 10: 2200-2208

66 Planas Vilà M. Nutritional and metabolic aspects of neurological diseases. Nutr Hosp 2014; 29: 3-12.

67 Kim HK, Park SK, Zhou JL, Taglialatela G, Chung K, Coggeshall RE et al. Reactive oxygen species (ROS) play an important role in a rat model of neuropathic pain. Pain 2004; 111: 116-124.
68 Burns SP, Golding DG, Rolle WA, Graziani V, Ditunno JF. Recovery of ambulation in motor- incomplete tetraplegia. Arch Phys Med Rehabil 1997; 78: 1169-1172.

69 Zaporozhan V, Ponomarenko A. Mechanisms of geomagnetic field influence on gene expression using influenza as a model system: basics of physical epidemiology. Int J Environ Res Public Health 2010; 7: 938-965.

70 Tabrah F, Hoffmeier M, Gilbert F Jr, Batkin S, Bassett CA. Bone density changes in osteoporosisprone women exposed to pulsed electromagnetic fields (PEMFs). J Bone Miner Res 1990; 5: 437-442.

71 Fanelli C, Coppola S, Barone R, Colussi C, Gualandi G, Volpe P et al. Magnetic fields increase cell survival by inhibiting apoptosis via modulation of $\mathrm{Ca} 2+$ influx. FASEB J 1999; 13: 95-102.

$72 \mathrm{Di}$ Carlo AL, White NC, Litovitz TA. Mechanical and electromagnetic induction of protection against oxidative stress. Bioelectrochemistry 2001; 53 87-95.

73 Milgram J, Shahar R, Levin-Harrus T, Kass PJ. The effect of short, high intensity magnetic field pulses on the healing of skin wounds in rats. Bioelectromagnetics 2004; 25: $271-277$. 